\title{
A Comprehensive Review on Pemphigus Vulgaris
}

\author{
Hina Handa ${ }^{1 *}$, Pooja Khare ${ }^{1}$ and Vijayta Sharva ${ }^{2}$ \\ ${ }^{1}$ Oral medicine Radiology, Peoples Dental Academy Bhopal, India \\ ${ }^{2}$ Department of Public Health Preventive Dentistry, Peoples Dental Academy Bhopal, India
}

Received: November 14, 2017; Published: November 30, 2017

*Corresponding author: Hina Handa, Senior Lecturer, MDS Oral Medicine Radiology, Peoples Dental Academy Bhopal, India; Email: hinahanda06@gmail.com

\begin{abstract}
Pemphigus vulgaris is a life threatening chronic autoimmune disease characterized by the formation of intraepithelial blisters on the skin and mucous membranes. Pemphigus vulgaris initially manifests in the form of intraoral lesions which spread to other mucous membranes and the skin. Pemphigus vulgaris (PV), the most common and important variant, is an autoimmune blistering disease characterized by circulating pathogenic IgG (immunoglobin) antibodies against desmoglein 3 (Dsg3), about half the patients also having Dsg1 autoantibody. Oral lesions are initially vesiculobullous but readily rupture, new bullae developing as the older ones rupture and ulcerate. Biopsy of perilesional tissue, with histological and immune staining examinations, is essential to the diagnosis. The diagnosis is based on clinical findings and laboratory analyses, and it is usually treated by the combined administration of corticosteroids and immune suppressants. Detection of the oral lesions can result in an earlier diagnosis. The current review focuses on the etiopathogenesis, diagnosis and current treatment of pemphigus vulgaris.
\end{abstract}

Keywords: Pemphigus; Oral mucosa; Autoimmune bullous disease

\section{Introduction}

Pemphigus vulgaris (PV) is a chronic autoimmune mucocutaneous diseases characterized by the formation of intraepithelial blisters. It is a rare disease (0.1-0.5 cases/100,000 inhabitants/yr), with onset usually in the fifth or sixth decade of life [1]. Various research have considered pemphigus as idiopathic in nature but strong evidences suggest it to be a disorder which is autoimmune in nature and is due to the presence of production of antibodies PV results from an autoimmune process in which auto antibodies are produced against the desmoglein protein which his adhesive protein and is responsible for holding the cells of epithelium together [2]. Dsg 3 is mainly present in the oral cavity while the desmoglin 1 is present over the skin region. The serum antibodies responsible for PV are always IgG type and these antibodies act against the desmoglein adhesion protein and lead to formation of bulla [1] (Table 1). The diagnosis of PV is based on three independent set of criteria: clinical features, histology, and immunological tests [3]. Research advances have expanded the therapeutic arsenal against PV, which now includes treatments with: pulse therapy (intravenous infusion of very high doses of immunosuppressant for a short time period); high doses of intravenous immunoglobulin; plasmapheresis; immunospecific immunoadsorption; extracorporeal photopheresis with exposure of serum to psoralens and UVA; antagonists of tumor necrosis factor a (TNFa); cholinergic antagonists; and anti- CD20 monoclonal antibodies (e.g., rituximab) [4].
However, no treatment has demonstrated superiority over the others Beutner [5]. The separation of cells, called acantholysis, takes place in the lower layers of the stratum spinosum, whereas according to Jordan et al it was noted that IgG auto antibodies are present in the sera which acts against the desmoglein protein which is responsible for cell to cell adhesion. Mahoney M et al. [6] said that the underlying mechanism responsible for causing the intraepithelial lesion of PV is the binding of IgG auto antibodies to desmoglein 3, a transmembrane glycoprotein adhesion molecule present on desmosomes. Some investigators believe that binding of the PV antibody activates proteases, whereas more recent evidence supports the theory that the PV antibodies directly block the adhesion function of the desmogleins. The separation of cells, called acantholysis, takes place in the lower layers of the stratum spinosum. Electron microscopic observations show the earliest epithelial changes as a loss of intercellular cement substance; this is followed by a widening of intercellular spaces, destruction of desmosomes, and finally cellular degeneration with ulceration. In a non immunologic reaction, a drug directly stimulates monocytes or lymphocytes to release cytotoxic chemical mediators. No immune response is involved in this situation, and these reactions are not antibody dependent.

\section{Investigation and Diagnosis}

Davenport S et al. [7] mentioned that diagnosis of pemphigus depends on biopsy confirmation of intraepithelial vesicle 
formation, acantholysis and the presence of Tzanck cells. Ettlin DA [8] discussed that demonstration of immunoglobulins especially IgG and complement in the intercellular space by direct immunofluorescence (DIF) is a very reliable test for pemphigus vulgaris. Scully [9] said that Indirect Immunofluorescence studies enable a search for circulating auto antibodies in the patient's serum and are usually performed after direct immunofluorescence studies reveal antibody deposits in the mucosa or skin. Bhol K et al. [10] reported that ELISA is a quantitative method for measuring antibody levels and a useful test for the diagnosis of pemphigus. This method is more sensitive and specific than IIF. It is also superior to sophisticated immunoblotting techniques. Davenport $\mathrm{S}$ et al. [7] entioned that diagnosis of pemphigus depends on biopsy confirmation of intraepithelial vesicle formation, acantholysis and the presence of Tzanck cells. Ettlin DA [8] discussed that demonstration of immunoglobulins especially IgG and complement in the intercellular space by direct immunofluorescence (DIF) is a very reliable test for pemphigus vulgaris. Scully [9] said that Indirect Immunofluorescence studies enable a search for circulating auto antibodies in the patient's serum and are usually performed after direct immunofluorescence studies reveal antibody deposits in the mucosa or skin. Bhol K et al. [10] reported that ELISA (enzyme linked immunosorbant assay) is a quantitative method for measuring antibody levels and a useful test for the diagnosis of pemphigus. This method is more sensitive and specific than indirect immunoflouroscence. It is also superior to sophisticated immunoblotting techniques.

\section{Management of Pemphigus Vulgaris}

Silverman S L et al. [11] proposed that treatment protocol for the initial/induction phase for PV typically involves the use of moderate to high dose(60 to $80 \mathrm{mg} /$ day in a single morning dose) corticosteroids and an adjunctive immunosuppressive drug, azathioprine, 100 to $150 \mathrm{mg} /$ day, for steroid-sparing effect, thereby reducing steroid-induced side effects. Moderate to high doses of corticosteroids administered daily, as a single dose, for 2 to 3 weeks are generally safe and well tolerated. Potential side effects of longterm steroid therapy have been extensively reviewed Tan-Lim R and Bystryn [12] stated that Plasmapheresis is the process by which plasma is removed from blood using a cell separator as antibodies are contained within plasma, plasmapheresis results in the removal of the pathogenic PV auto antibodies. Multiple case series have evaluated the efficacy of plasmapheresis in treating PV.20-23 of the 28 patients evaluated in these studies, 18 (64\%) experienced complete remission, 6 (33\%) experienced partial remission and 4 (22\%) had no clinical improvement. Adverse effects encountered included systemic infections, acute hepatitis, thrombocytopenia, anemia, hypocalcemia, nausea, dizziness, urticaria, fever, and hypotension. In a multicentric study $(n=40)$ were randomized to receive prednisolone alone or prednisolone plus large-volume plasma exchange. While plasmapheresis failed to demonstrate a therapeutic benefit in this study, it has been suggested that an additional immunosuppressive (i.e, cyclophosphamide) or immunomodulatory (i.e., IVIg) therapy may be required to prevent the rebound production of pathogenic auto antibodies associated with disease flares. High dose intravenous immunoglobulin (IVIg) has been reported to be of benefit as a steroid-sparing agent in the management of patients with PV. In a review of eight reports high dose IVIg was effective in 17 of 18 patients.Cyclophosphamide is an alkylating cytotoxic agent that is a highly effective steroid sparing agent. In a review of five studies with a total of 51 patients, complete remission was achieved in $32 \%$ of patients. The rate of remission for each of the five studies that were reviewed was very wide (0\%-80\%), reflecting the small number of patients in each study. Cyclophosphamide has been used as pulse therapy $(1 \mathrm{~g}$ intravenously every four weeks) [12].

Rook AH et al. [13] mentioned that Extracorporeal photo chemotherapy [ECP] is a new form of immunotherapy which involves the extracorporeal photo inactivation of peripheral blood cells by 8-methoxypsoralen in the presence of ultraviolet A irradiation, followed by re administration of the cells. There are only 2 small case series and 2 case reports in the literature that document the use of ECP for refractory PV. Of the 9 PV patients treated with ECP in these studies, all experienced significant clinical improvement, while no adverse effects from ECP were noted. Shimanovich I et al. [14] said that Immunoadsorption resulted in a dramatic clinical response and a rapid decline in desmoglein-specific IgG auto antibodies. Recently, a small case series demonstrated that IA, administered in combination with rituximab, may result in long-term remission. In all studies, IA was safe and well tolerated. Harman KE et al. [15] concluded that systemic corticosteroids are still the most frequently used drugs and best-established therapy in pemphigus. The guidelines for managementof PV by the British Association of Dermatologists recommend patients with mild disease to receive an initial prednisolone dosage of 40 to $60 \mathrm{mg} / \mathrm{d}$ and in more severe cases, 60 to $100 \mathrm{mg} / \mathrm{d}$. If no response is achieved within 1 week, the dosage is increased by $50 \%$ to $100 \%$ until disease control. Yeh SW et al. [16] reported that adjuvant drugs are usually administered in combination with systemic corticosteroids so as to reduce corticosteroid related side effects and increase efficacy. Conventional adjuvants include various immunosuppressive agents such as azathioprine, mycophenolate mofetil, methotrexate, cyclophosphamide, chlorambucil and cyclosporine, as well as antiinflammatory agents such as gold, dapsone, colchicine and a variety of tetracycline antibiotics (Table 1).

Table 1: Drug Regimes in Management of Pemphigus Vulgaris.

\begin{tabular}{|c|c|c|c|}
\hline \multirow{2}{*}{ Drug Type } & Systemic Agent & Mode of Administration & Dose \\
\hline \multirow{3}{*}{ Systemic corticosteroid } & Prednisolone & Oral & \multirow{2}{*}{$1-2 \mathrm{mg} \backslash \mathrm{kg} \backslash \mathrm{d}$} \\
\cline { 2 - 3 } & Dexamethasone & Oral or IV Pulse & $50-200 \backslash \mathrm{d}$ for 3-5 day \\
\cline { 2 - 3 } & Methyl prednisone & IV Pulse & \\
\cline { 2 - 3 } & &
\end{tabular}




\begin{tabular}{|c|c|c|c|}
\hline \multirow{4}{*}{$\begin{array}{c}\text { Immunosuppressive and Ant } \\
\text { inflammatory Agents }\end{array}$} & Azathioprine & Oral & $3-4 \mathrm{mg} \backslash \mathrm{kg} \backslash$ day \\
\cline { 2 - 4 } & Chlorambucil & Oral & $6-9 \mathrm{mg} \backslash \mathrm{d}$ \\
\cline { 2 - 4 } & Cyclophosphasmide & Oral & $20 \mathrm{mg} \backslash \mathrm{d}$ \\
\cline { 2 - 4 } & Cyvlosporine & Oral & $5-30 \mathrm{mg} \backslash \mathrm{wk}$ \\
\cline { 2 - 4 } & Dapsone & Oral & $100-200 \mathrm{mg} \backslash \mathrm{d}$ \\
\cline { 2 - 4 } & Erythromycin & Oral & $1,200 \mathrm{mg} \backslash$ day \\
\cline { 2 - 4 } & Gold & IM Injection & $25-50 \mathrm{mg} \backslash$ weekly \\
\cline { 2 - 4 } & Leflunomide & Oral & $50-200 \mathrm{mg} \backslash$ day \\
\cline { 2 - 4 } & Minocycline & Oral & $20 \mathrm{mg} \backslash \mathrm{d}$ \\
\cline { 2 - 4 } & Mycophenolate & Oral & $25-50 \mathrm{mg}$ biweekly \\
\hline
\end{tabular}

Bystryn JCetal. [17] discussed that Intravenous Immunoglobulin (IVIg) is a fractionated and purified blood product derived from the plasma of between 1,000 and 15,000 healthy donors per batch. It contains a high concentration of IgG and has a broad range of antibodies directed against pathogens, foreign antigens, and selfantigens. Although its exact mechanism of action remains unclear, IV IgG is associated with a rapid and selective decline in the serum levels of pathogenic PV auto antibodies. Jacobi A et al. [18] stated that TNF-á antagonists, tumor necrosis factor may be beneficial for the treatment of PV as experimental studies have demonstrated that TNF-á plays a role in the acantholytic process. Two case reports document the successful use of Infliximab for refractory PV. Anhalt G et al. [19] concluded that selective therapy using Intravenous Desmoglein 3 peptides was developed to suppress the production of anti-desmoglein 3 antibodies through inactivation and/or deletion of disease-associated CD4+ T lymphocytes. Joly P et al. [20] mentioned that Rituximab is a chimeric murine/human IgG1 anti-CD20 monoclonal antibody that targets pre-B and mature B lymphocytes, resulting in complement and antibody-dependent cytotoxicity and apoptosis. Multiple case reports suggest that rituximab is an effective treatment option for PV. The largest clinical study evaluating the use of rituximab in PV has been a case series of 14 patients with refractory PV in which 12 (86\%) experienced a complete remission at 3 months after a single cycle of rituximab.

Schmidt E et al. [21] concluded that Rituximab is a chimeric murine/human IgG1 anti-CD20 monoclonal antibody that targets pre-B and mature B lymphocytes, resulting in complement and antibody-dependent cytotoxicity and apoptosis. Rituximab reduces circulating B cells, thereby preventing their maturation into antibody-producing plasma cells. Of the 18 patients with refractory PV reviewed, 3 (17\%) experienced complete remission, 4 (22\%) experienced clinical remission with further therapy required and 11 (61\%) experienced partial remission.

\section{Conclusion}

The diagnosis of PV at an early stage when it is limited to oral cavity requires careful assessment and correlation of the clinical appearance, histological features and immunofluroscence findings. Their exist various treatment modality for treating Pemphigus which may range from topical and systemic steroid to current immunomodulators. The incidence of remissions in pemphigus is unclear, because these are usually reported at a single point in the evolution of the disease. Thus, it is uncertain whether treatment simply suppresses the manifestations of the disease and consequently must be continuously administered, or induces complete and long-lasting remissions that permit therapy to be discontinued. The prognosis of untreated oral lesions is a progression that involves other mucosa, including the skin. When treated, the prognosis depends on the age of the patient, the initial severity, the extent of lesions, the interval between symptom onset and start of treatment, and the drug dose required to control the disease, among other factors.

\section{References}

1. Ruocco E, Baroni A, Wolf R, Ruocco V (2005) Life- threatening bullous dermatoses: pemphigus vulgaris. Clin Dermatol 23: 223-226.

2. Bystryn JC, Rudolph JL (2005) Pemphigus. Lancet 366: 61-73.

3. Bascones-Martínez A, Figuero-Ruiz E, Esparza-Gómez GC (2005) [Oral ulcers]. Med Clin (Barc) 125(15): 590-597.

4. Prajapati V, Mydlarski PR (2008) Advances in pemphigus therapy. Skin Therapy 13: 4-8.

5. Ernst H Beutner, Lever WF, Witebsky E, Jordon R, Chertock B (1965) Autoantibodies in Pemphigus Vulgaris Response to an Intercellular Substance of Epidermis. JAMA 192: 682-688.

6. Mahoney MG, Aho S, Uitto JR, Stanley JR (1998) The members of the plakin family of proteins recognized by paraneoplastic pemphigus antibodies include periplakin. J Invest Dermatol 111(2): 308-313.

7. Davenport S, Chen SY, Miller AS (2001) Pemphigus vulgaris: clinicopathologic review of 33 case in the oral cavity. Int J Periodontics Restorative Dent 21(1): 85-90.

8. Ettlin DA (2005) Pemphigus vulgaris: update on etiopathogenesis, oral manifestations, and management. Dent Clin North Am 49: 107-125.

9. Crispian Scully (2002) Pemphigus Vulgaris: Update on etiopathogenesis, oral manifestations, and management. Crit Rev Oral Biol Med 13: 397408.

10. Bhol K, Tyagi S, Natarajan K, Nagarawalla N, Ahmed AR (1998) Use of recombinant pemphigus vulgaris antigen in development of ELISA and IB assays to detect pemphigus vulgaris autoantibodies. J Eur Acad Dermatol Venereol 10(1): 28-35.

11. Silverman S, Gorsky M, Lozada-Nur F, Liu F (1986) Oral mucous membrane pemphigoid. Oral Surg Oral Med Oral Pathol 61: 233-237.

12. Tan-Lim R, Bystryn JC (1990) Effect of plasmapheresis therapy on circulating levels of pemphigus antibodies. J Am Acad Dermatol 22(1): $35-40$. 
13. Rook AH, Jegasothy BV, Heald P, Nahass GT, Ditre C, et al. (1990) Extracorporeal photochemotherapy for drug-resistant pemphigus vulgaris. Ann Intern Med 112(4): 205-303.

14. Shimanovich I, Herzog S, Schmidt E, Klinker E, Brocker EB, et al. (2006) Improved protocol for treatment of pemphigus vulgaris with protein $A$ immunoadsorption. Clin Exp Dermatol 31(6): 768-774.

15. KE Harman, S Albert, MM Black (2003) Guidelines for the management of pemphigus vulgaris. British Journal of Dermatology 149: 926-937.

16. Yeh SW, Sami N, Ahmed AR (2005) Treatment of pemphigus vulgaris: current and emerging options. Am J Clin Dermatol 6(5): 327-342.

17. Bystryn JC, Jiao D, Natow S (2002) Treatment of pemphigus with intravenous immunoglobulin. J Am Acad Dermatol 47(3): 358-363.
18. Jacobi A, Shuler G, Hertl M (2005) Rapid control of therapy-refractory pemphigus vulgaris by treatment with the tumour necro sis factor-alpha inhibitor infliximab. Br J Dermatol 153(2): 448-449.

19. Anhalt G, Werth V, Strober B (2005) An open-label phase I clinical study to assess the safety of PI-0824 in patients with pemphigus vulgaris. J Invest Dermatol 125: 1088.

20. Joly P, Mouquet H, Roujeau JC, Michel D, D Gilbert, et al. (2007) A single cycle of rituximab for the treatment of severe pemphigus. N Eng J Med 357: 545-552.

21. Schmidt E, Hunzelmann N, Zillikens D (2006) Rituximab in refractory autoimmune bullous diseases. Clin Exp Dermatol 31(4): 503-508.

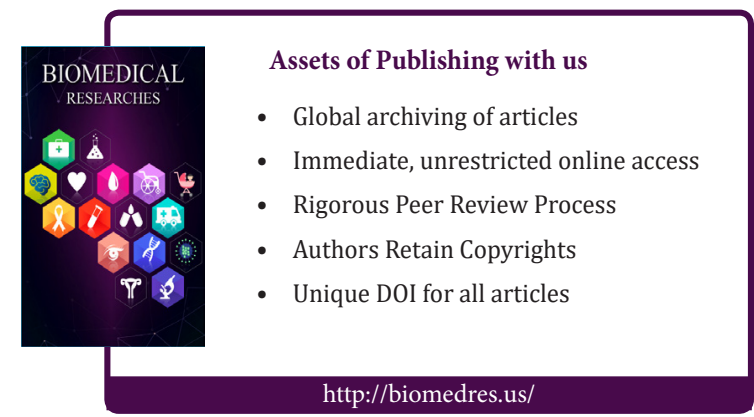

